\title{
Pregnancy with portal hypertension: a case report
}

\author{
Mansi Dhingra, Syed Nawaz Ahmad* \\ Department of Obstetrics and Gynecology, ESI PGIMSR, Basaidarapur, New Delhi, India
}

Received: 08 January 2018

Accepted: 03 February 2018

\section{*Correspondence:}

Dr. Syed Nawaz Ahmad,

E-mail: dr.syednawaz@gmail.com

Copyright: $\odot$ the author(s), publisher and licensee Medip Academy. This is an open-access article distributed under the terms of the Creative Commons Attribution Non-Commercial License, which permits unrestricted non-commercial use, distribution, and reproduction in any medium, provided the original work is properly cited.

\begin{abstract}
Even though pregnancy is rare with cirrhosis and advanced liver disease, it may co-exist in the setting of noncirrhotic portal hypertension as liver function is preserved. When encountered together it presents a complex clinical dilemma. Physiological hemodynamic changes associated with pregnancy, needed for meeting demands of the growing fetus, worsen the portal hypertension thereby putting mother at risk of potentially life-threatening complications like variceal hemorrhage. Pregnancy also predisposes the patient to develop hepatic decompensation. Thus, management of such cases requires multidisciplinary approach involving obstetricians experienced in dealing with high risk cases, hepatologists, anesthetists and neonatologists. Here through the case report of a 23 year old primigravida with Non Cirrhotic portal hypertension with 33 weeks gestational age in preterm labor we intend to focus upon different aspects of pregnancy with portal hypertension, its complications and management strategies.
\end{abstract}

Keywords: Cirrhosis, Pregnancy, Portal hypertension

\section{INTRODUCTION}

Pregnancy is a normal physiological state characterized by numerous hemodynamic changes. These hemodynamic changes, although necessary for a normal pregnancy, pose a special problem in the presence of portal hypertension.

In developed countries, cirrhosis of liver is the most common cause of portal hypertension. Although rare, the occurrence of pregnancy is not altogether unknown in this population. This is caused by reduced fertility as a consequence of anovulatory cycles, altered endocrine metabolism, and a relatively older age of these patients. ${ }^{1,2}$

In developing countries, other causes like extrahepatic portal vein obstruction contribute significantly to noncirrhotic portal hypertension $(\mathrm{NCPH})$. In patients with noncirrhotic portal hypertension $(\mathrm{NCPH})$ the hepatic synthetic functions are relatively well preserved and so is fertility. 3 Pregnancy in a patient with portal hypertension is a unique problem that needs specialized care to prevent potentially life-threatening complications such as gastrointestinal hemorrhage. It is therefore important to understand the effect of pregnancy on portal hypertension and vice versa so that untoward incidents like fetal morbidity, mortality and gastrointestinal hemorrhage can be avoided.

\section{CASE REPORT}

A 23 year old patient, primigravida with 33 weeks gestational age, known case of non-cirrhotic portal hypertension reported in labour room in preterm labour.

The patient gave history of 2 episodes of hematemesis 6 months prior to conception. Endoscopy done preconceptionally showed grade 3 varices, ultrasonography done at that time showed hepatomegaly without distortion of liver architecture, massive splenomegaly, dilatation of portal collaterals, absence of 
ascites and a single live intrauterine gestation corresponding to the gestational age.

In the present pregnancy, there was no history bleeding tendencies, jaundice. liver function tests, coagulation profile were normal. Markers of infective hepatitis were negative . Pancytopenia was present.

Patient was allowed for spontaneous progress of labour, started on antenatal corticosteroids, Tab propranolol 20 mg three times a day (after consultation with the gastroenterologists). High risk consent was taken in view of prematurity, risk of variceal bleeding, pancytopenia, need of blood and blood products. Adequate amount of blood and plasma were arranged. The second stage of labour was cut short by instrumental delivery. Patient delivered a $1.5 \mathrm{~kg}$ male baby that cried immediately after birth and was shifted to nursery for observation. Third stage of labour was managed actively. Postpartum recovery was uneventful.

\section{DISCUSSION}

In pregnant women, alcoholic cirrhosis is uncommon while viral or autoimmune related cirrhosis is more common in developing countries. The non-cirrhotic causes of portal hypertension include extra-hepatic portal vein obstruction, non cirrhotic portal fibrosis, portal vein thrombosis, Budd-Chiari syndrome, infection or congenital hepatic fibrosis. ${ }^{6}$

Numerous hemodynamic and physiological changes occur during pregnancy as an adaptation to the needs of the growing fetus. These changes start as early as six weeks and peak around 32 weeks. One of the earliest changes is an increase in plasma output by $40-50 \%$. Maternal cardiac output increases by $30-50 \%$ due to increase in stroke volume and the heart rate. There is decline in systemic vascular resistance as a result of progesterone effect and development of placental vascular bed. As a result of all of these changes, there is a profound alteration in systemic hemodynamics resulting in a hyperdynamic state with increased pulse pressure. These changes can worsen the portal hypertension in pregnant patients with portal hypertension and markedly increase the risks of variceal hemorrhage. The pregnant woman has a $20-27 \%$ chance of esophageal bleed which increases markedly in case she has demonstrable varices. ${ }^{5}$ Active variceal bleeding may occur at all stages of the pregnancy through second and third trimester, risk of variceal bleeding being the maximum in $2^{\text {nd }}$ stage of labor.

Prognosis of portal hypertension during pregnancy depends upon the underlying cause and the extent of derangement of liver function. Maternal prognosis is better with noncirrhotic portal hypertension and poor with cirrhosis of the liver. Maternal mortality ranges between $2 \%$ and $18 \%$; being maximum with cirrhosis.
The causes of death are generally hematemesis, hepatic coma or postpartum hemorrhage. ${ }^{2}$ The mother is also at risk of developing severe anemia, splenic artery aneurysm rupture, ascites, spontaneous bacterial peritonitis. Perinatal mortality ranges between $11 \%$ and $18 \%$, mainly due to preterm delivery or intrauterine growth restriction (IUGR).

Extensive and detailed pre-conceptional counseling, evaluation and antenatal and perinatal monitoring is needed in patients with portal hypertension with or without cirrhosis planning pregnancy. Varices should be tackled prior to planning a pregnancy, endoscopic variceal ligation is the preferred therapy and nonresponders should be offered surgery in the form of shunt procedure or splenectomy. Drugs should be reviewed for adverse effects on the fetus and alternative safe drugs to be changed, and also dose needs to be tailored. Selective beta -blockers can be continued as their benefits outweigh risks. Medical termination of pregnancy may be advised in case of severe hepatic decompensation like ascites, encephalopathy, and liver failure.

Antenatal management requires strict maternal and fetal monitoring by a multi- disciplinary team. The routine antenatal management should be given with special watch out for the potential complications like variceal bleed and liver failure. Liver function and hematological assessment should be done 4 weekly, fetal growth needs to be monitored vigilantly and effects of the drugs need to be watched. Close maternal and fetal monitoring is recommended two weekly.

Since variceal bleed is the single important complication linked with poor pregnancy outcome, the basic aim is to prevent it. This can be done by assessment and tackling the varices prior to planning a pregnancy. In cases of unplanned pregnancy, proper risk assessment should be performed. Endoscopy is the gold standard to assess the risk of bleeding in patients with esophageal varices. Upper gastrointestinal endoscopy is safe during pregnancy, the main risk being fetal hypoxia due to sedation or positioning. ${ }^{7}$ Current American Association for the Study of Liver Disease (AASLD) recommendations include screening endoscopy in the second trimester as that is the time of maximum increase in the portal pressure. ${ }^{8}$

The treatment options in presence of esophageal varices are both medical and surgical. Non-selective beta blockers used to reduce portal pressure also reduce the risk of first bleed by half but the principal risk of using them in pregnancy is fetal growth restriction and fetal bradycardia. Endoscopic variceal ligation of the large varices can also be done during pregnancy to prevent variceal bleeding. Current literature (Baveno V consensus workshop) recommends EVL for acute esophageal variceal bleed, although, endoscopic sclerotherapy may be used if banding is technically difficult. ${ }^{9}$ 
Pregnancy can be allowed to go to term if the disease is well compensated. Early termination of pregnancy may be warranted in case of any obstetrical indication or progressive liver failure. In case of planned termination before 34 weeks, antenatal corticosteroids can be administered for fetal lung maturity. There are no recommendations as to the preferred mode of deliveryvaginal versus caesarean section in patients with portal hypertension.

The management during labor needs to be individualized depending on cause of portal hypertension and the disease status. Adequate amount of blood and plasma should be arranged and measures for balloon tamponade for the variceal hemorrhage must be handy. Second stage of labor may be shortened prophylactically to avoid overstraining by the mother. ${ }^{10}$ The third stage should be managed actively; methergin should be avoided amongst the oxytocics. Postpartum hemorrhage should be anticipated and managed vigilantly.

\section{CONCLUSION}

Pregnancy with portal hypertension may be associated with adverse maternal and perinatal outcome especially in cirrhotic portal hypertension but pregnancy is not contra-indicated as was once believed. The management of pregnancy with portal hypertension should only be done at tertiary care centres by a multidisciplinary team with backup facilities for intensive care and blood transfusion.

Funding: No funding sources

Conflict of interest: None declared

Ethical approval: Not required

\section{REFERENCES}

1. Britten RC. Pregnancy and esophageal varices. Am J Surg 1982;143(4):421-5.
2. Cheng YS. Pregnancy in liver cirrhosis and/or portal hypertension. Am J Obstet Gynecol. 1977;128(7):812-22.

3. Pajor A, Lehoczky D. Pregnancy and extrahepatic portal hypertension. Review and report on the management. Gynecol Obstet Invest. 1990;30(4):193-7.

4. Thompson E, Williams R, Sherlock S. Liver function in extra-hepatic portal obstruction. Lancet. 1964;ii:1352-6.

5. Lopez-Mendes Eric. Lourdes Avila-Escobedo. Pregnancy and portal hypertension a pathology view of physiologic changes. Ann Hepatol. 2006;5:21923.

6. Sandhu BS, Sanyal AJ. Pregnancy and liver disease. Gastroenterol Clin North Am. 2003;32:407-36.

7. O'Mahony S. Endoscopy in pregnancy. Best Pract Res Clin Gastro- enterol. 2007;21:893-9.

8. Garcia-Tsao G, Sanyal AJ, Grace ND, Carey W, Practice Guidelines Committee of the American Association for the Study of Liver Diseases, Practice Parameters Committee of the American College of Gastroenterology. Prevention and management of gasteroeso- phageal varices and variceal hemorrhage in cirrhosis. Hepatology. 2007;46:922-38.

9. deFranchis R. Baveno V faculty. Revising consensus in portal hyper- tension: report of Baveno $\mathrm{V}$ consensus workshop on methodology of diagnosis and therapy in portal hypertension. J Hepatol. 2010;53:762-8.

10. Heriot JA, Steven CM, Sattin RS. Elective forceps delivery and extra- dural anesthesia in a primigravida with portal hypertension and esophageal varices. $\mathrm{Br} \mathbf{J}$ Anes. 1996;76:325-7.

Cite this article as: Dhingra M, Ahmad SN.

Pregnancy with portal hypertension: a case report. Int J Reprod Contracept Obstet Gynecol 2018;7:3896-8. 